\title{
Linx
}

Revue des linguistes de l'université Paris X Nanterre

48 | 2003

Approches syntaxiques contemporaines

\section{Les constructions infinitives dans les grammaires d'arbres polychromes hiérarchisées}

Anne Lablanche

\section{(2) OpenEdition}

1 Journals

Édition électronique

URL : http://journals.openedition.org/linx/119

DOI : $10.4000 /$ linx. 119

ISSN : 2118-9692

Éditeur

Presses universitaires de Paris Nanterre

Édition imprimée

Date de publication : 1 juin 2003

Pagination : 29-42

ISBN : 0246-8743

ISSN : 0246-8743

Référence électronique

Anne Lablanche, "Les constructions infinitives dans les grammaires d'arbres polychromes

hiérarchisées », Linx [En ligne], 48 | 2003, mis en ligne le 01 octobre 2003, consulté le 10 décembre

2020. URL : http://journals.openedition.org/linx/119; DOI : https://doi.org/10.4000/linx.119 


\title{
Les constructions infinitives dans les grammaires d'arbres polychromes hiérarchisées*
}

\author{
Anne Lablanche, Université Paris $X$ - Nanterre, \\ CNRS-MoDyCo, \\ anne.lablanche@u-paris10.fr
}

Dans cet article, nous proposons une analyse syntaxique des constructions infinitives (ou infinitives) en français. Pour nous, suivant en cela Huot (1981) et Baschung (1991), une construction infinitive est le groupe formé par un verbe infinitif et son environnement. Cette définition est plus large que celle de Grevisse (1964) et Lorian (1962), et que celle proposée plus récemment par Legrand (1999) et Leeman (2002). Les premiers considèrent que l'on peut parler de proposition infinitive quand le verbe à l'infinitif est introduit par un outil de subordination, relatif ou interrogatif. Et lorsque son sujet, exprimé ou non, est différent du verbe principal. Quant aux seconds, ils remettent en cause le fait d'appeler "proposition infinitive » Paul partir dans je vois Paul partir, car la séquence ne possède pas les propriétés d'un constituant.

Les constructions infinitives, dans une phrase, peuvent apparaître à la suite d'un nom (la faculté de parler est une propriété), d'un verbe (Jean rêve de partir), d'un adjectif (Jean est content de venir). Nous nous limitons au cas où la construction infinitive est sous-catégorisée par un verbe fini. Nous considérons trois sous-cas : lorsque le verbe au mode infinitif suit immédiatement un verbe conjugué, lorsqu'il est introduit par l'item de ou l'item à, et lorsqu'il est introduit par une autre préposition (pour, sans,...). La question se posera pour certaines constructions de savoir s'il faut les considérer comme des groupes prépositionnels ou pas.

Les grammaires d'arbres polychromes hiérarchisées (Cori et Marandin, 1998) forment le cadre dans lequel sera analysée la construction infinitive. Ce formalisme syntaxique repose sur trois caractéristiques essentielles. En premier lieu, il fait sienne l'hypothèse positionnelle (Milner 1989) selon laquelle il faut distinguer une position de ce qui occupe cette position. Dans les arbres polychromes, une position est représentée par une « couleur». En deuxième lieu, une « relation d'interdépendance »,

\footnotetext{
* Je remercie A. Delaveau, D. Leeman, M. Cori et J.M. Marandin pour leurs commentaires et suggestions.
} 
associée aux arbres, permet de rendre compte des phénomènes qui ne peuvent pas être traités à l'aide du mécanisme classique de l'unification. En troisième lieu, ces grammaires sont hiérarchisées, c'est-à-dire qu'elles supposent que les structures élémentaires d'une langue forment des classes, et que l'on peut exprimer des règles générales qui opèrent sur ces classes.

\section{Les grammaires d'arbres polychromes hiérarchisées}

\subsection{La notion de position}

Les grammaires d'arbres polychromes (GAP, Cori et Marandin, 1993) ont été inspirées par l'hypothèse positionnelle de Milner (1989). La notion de position, dans le cadre des GAP, est très proche de la notion de site proposée par Milner. Les positions sont définies indépendamment de la catégorie des constituants qui les occupent. Il y a une organisation des positions qui rend compte du placement des constituants les uns par rapport aux autres dans les énoncés. Les constituants d'une structure se disposent par rapport à une position privilégiée : la position noyau qui donne au terme qui l'occupe le rôle de pivot.

Les GAP utilisent la représentation arborescente pour rendre compte des configurations décrites. Un arbre se définit comme un ensemble de nœuds reliés par des branches. Dans un arbre polychrome, chaque branche possède une couleur. Pour le français, cinq positions ont été définies, autrement dit cinq couleurs, représentées par les nombres de 1 à $5^{1}$.

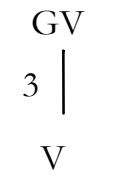

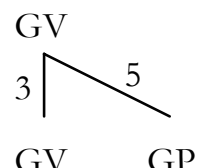

GV

b

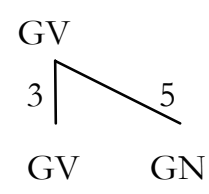

c

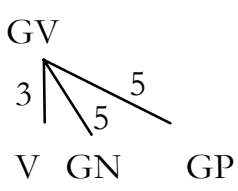

d

Figure 1

La couleur 3 représente la position pivot. Les couleurs permettent de distinguer les branches dominées par une même catégorie. GV, V, GN, et GP sont des étiquettes catégorielles. Toute position est définie en fonction de la catégorie qui la domine. L'attribution d'une même couleur à plusieurs branches à un même niveau dans l'arbre permet d'exprimer le fait qu'une position est occupée par plusieurs constituants à la fois (fig. 1d). Autrement dit dans Jean donne un bonbon à Marie, le GN un bonbon et le GP à Marie sont tous deux des compléments et ont la même position, ici la position 5 .

Pour décrire l'analyse d'une catégorie, on utilise un arbre polychrome de profondeur 1, dans lequel il n'y a pas plus d'une branche d'une couleur donnée : un tel arbre est appelé arbre élémentaire.

\footnotetext{
${ }^{1}$ Les positions 2 et 4 ne sont pas illustrées dans le cadre de cet article. Elles servent, par exemple, à l'analyse des constituants incidents, parmi lesquels figurent les incises.
} 
Les grammaires sont composées d'un ensemble d'arbres élémentaires, tels qu'en figure 1 les arbres a, b et c. Un arbre élémentaire peut également être représenté sous la forme d'une règle :
(a) $\mathrm{GV} \rightarrow 3 \mathrm{~V}$
(b) GV $\rightarrow 3$ GV 5 GP
(c) GV $\rightarrow 3$ GV 5 GN

L'arbre 1d n'est pas un arbre élémentaire. Il est le résultat de la composition des arbres 1a, 1b et 1c. La composition, qui s'accompagne dans certains cas d'un « compactage », est une opération qui permet de construire des arbres plus complexes à partir d'arbres. Ainsi, l'arbre 2a (ci-dessous) est obtenu par composition de l'arbre 1b et de l'arbre $1 \mathrm{a}$.

Le compactage, dans les GAP, permet la réduction des niveaux d'un arbre. Cette opération s'effectue selon des conditions bien spécifiques. Le compactage s'applique obligatoirement après la composition lorsqu'on obtient un arbre dans lequel deux nœuds de même catégorie sont reliés par une branche de couleur 3 (arbre 2a) Cette même branche est alors supprimée et les deux nœuds fusionnent (arbre 2b).

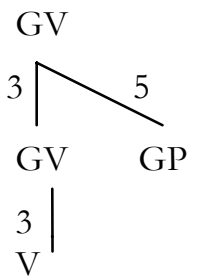

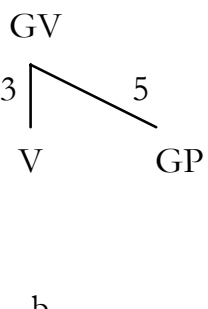

Figure 2

Un arbre obtenu par composition peut à son tour être composé avec un autre. Ainsi, on peut obtenir des représentations de phrase complète comme en figure 7 (voir ci-après, \2.2).

\subsection{Traits et interdépendance}

Les GAP possèdent un système de traits qui permet de régler plus précisément la combinatoire des unités. Ces traits sont associés aux nœuds des arbres. Certains traits admettent comme valeur une variable ${ }^{2}$. Une caractéristique originale des GAP est l'introduction de relations d'interdépendance associées aux arbres, qui permettent de généraliser certaines représentations par des règles uniques, tout en ne manquant pas les différenciations de détail. Les relations d'interdépendance des arbres élémentaires se combinent au moment de la composition pour définir une relation générale qui contraint l'ensemble des traits apparaissant dans l'arbre composé.

\footnotetext{
${ }^{2}$ On note les variables de traits par des majuscules et les constantes par des minuscules.
} 
L'utilisation des traits et des variables (de traits) permet de traiter par exemple la sous-catégorisation des verbes. Les différents compléments sous-catégorisés peuvent occuper la même position. A chaque type de complément (GN, GP, GS 3 ...) est associé un trait qui porte la spécification oui si un complément est attendu, non si le complément n'est pas attendu ou a déjà été trouvé. Le trait a1 vaut pour le complément direct, a2 pour l'indirect et a0 pour le sujet, qui est considéré comme un type de complément.

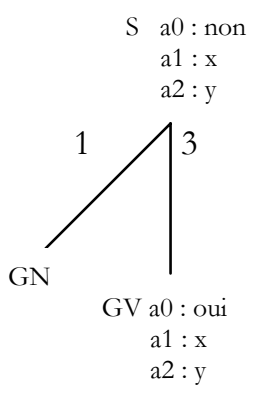

A

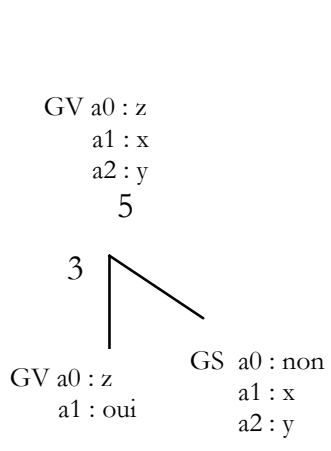

b

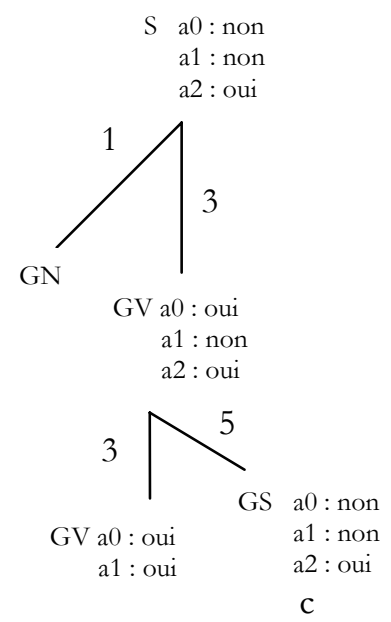

Figure 3

Les arbres $3 \mathrm{a}$ et $3 \mathrm{~b}$ sont des arbres élémentaires de la grammaire. L'arbre $3 \mathrm{a}$ représente une phrase standard, c'est-à-dire constituée d'un GN et d'un GV. L'arbre 3b représente le cas d'un GS complément dans un GV. Par composition, on obtient l'arbre $3 c$ représentant par exemple la phrase Jean promet qu'il viendra. C'est-à-dire qu'en $3 c$, le nœud GV qui correspond à promet attend un complément direct : a1 est marqué oui. Au niveau supérieur, d'après l'arbre élémentaire $3 \mathrm{~b}$, la transmission du trait a1 ne vient pas du GV mais du GS. Dans ce même GV, a0 est marqué oui car le verbe attend son sujet. Au nœud $\mathrm{S}$, d'après $2 \mathrm{a}$, a0 prend la valeur non parce que le sujet est réalisé.

Dans certains cas, la combinatoire des traits est définie par une relation d'interdépendance. La relation se présente comme une liste d'assignations possibles de valeurs à l'ensemble des variables de l'arbre. A titre d'illustration, prenons le cas des relatives et des complétives pour lequel l'interdépendance autorise un traitement unifié. La règle utilisée est :

(d) gs [a0: X, a1: Y, a2: Z] $\rightarrow 1$ COMP [type: T] 3 s [mode: M, a0: X, a1: Y, a2: Z]

\footnotetext{
${ }^{3}$ Le GS peut être soit une relative soit une complétive.
} 
COMP est l'étiquette générique des complémenteurs (pronoms relatifs, conjonctions de subordination et, comme on le verra, certaines prépositions). X, Y et $\mathrm{Z}$ sont des variables qui peuvent prendre les valeurs oui ou non. Quant à la variable $\mathrm{T}$, elle se réalise en que, qui ou dont selon les cas. Mode est un attribut qui indique si le verbe est à l'infinitif, l'indicatif ou encore au subjonctif.

Cette règle est accompagnée de la relation d'interdépendance qui met en jeu quatre attributs : le type du COMP (dont la valeur est T), a0, a1 et a2.

\begin{tabular}{|c|l|l|l|}
\hline T & X & Y & Z \\
\hline que & non & - & - \\
\hline qui & oui & - & - \\
\hline qui & non & - & - \\
\hline dont & non & - & oui \\
\hline
\end{tabular}

Lorsque le type est que ${ }^{4}$, a 0 correspondant au sujet a obligatoirement la valeur non. Cela permet de refuser *la pomme que mange. Mais a1 et a2 qui correspondent aux compléments sont indéterminés 5 .

Pour le GS de type qui, il y a deux possibilités. Pour la première, a0 a la valeur oui, a1 et a2 sont indéterminés. Cela permet de refuser *la pomme qui Jean mange. La seconde correspond aux phrases telles que Je me demande qui tu as vu.

En revanche, pour le type dont, a2 a la valeur oui. Il est nécessaire qu'un complément indirect soit possible car on doit refuser *la pomme dont Jean mange.

En figure 4, est présenté l'arbre de Jean pense que Marie viendra, dans lequel la relation d'interdépendance intervient. Le nœud GV correspondant à viendra a «a0 : oui » car un sujet est attendu. En revanche, le nœud S, correspondant à Marie viendra, possède un sujet $(\mathrm{a} 0$ : non). Ce trait est transmis au niveau supérieur (au GS). La relation d'interdépendance a permis la combinaison du COMP que avec le S Marie viendra, elle aurait interdit * Jean pense que viendra.

\footnotetext{
${ }^{4}$ Que est à la fois la conjonction de subordination et le pronom relatif qui ne sont pas différenciés ici. Cela permet de traiter les dépendances à distances, comme par exemple dans La femme que Paul croit que Jean fréquente.

${ }^{5}$ C'est-à-dire qu'ils peuvent prendre la valeur oui ou la valeur non. Dans le tableau, l'indétermination est notée -.
} 
Anne Lablanche

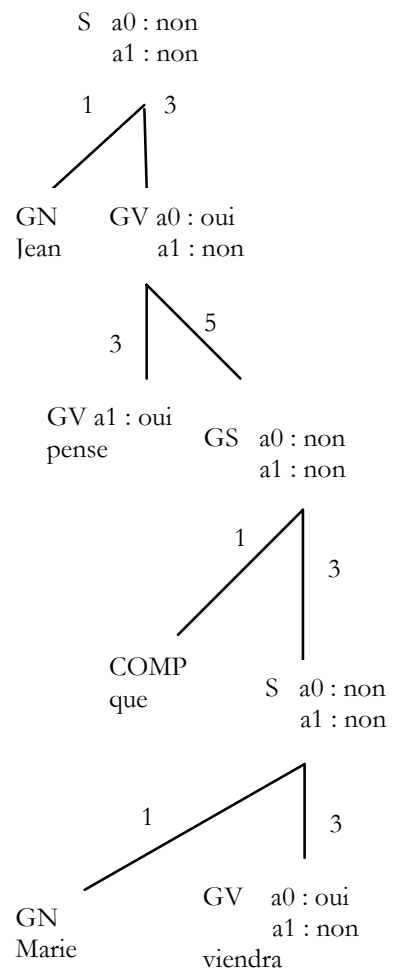

Figure 4

\subsection{La biérarcbisation}

Depuis les années 1980, les formalismes syntaxiques intègrent une organisation hiérarchique afin de structurer les connaissances linguistiques (lexicales, phonologiques, syntaxiques, sémantiques) (cf. Daelmans et al., 1992) et de dégager des généralisations. La relation d'héritage permet de rendre plus concises les descriptions linguistiques et a été utilisée essentiellement pour l'organisation lexicale.

Les GAP ont été, elles aussi, structurées hiérarchiquement, et sont devenues les GAPH (Cori et Marandin, 1998). Les informations sont indiquées au niveau adéquat de la hiérarchie. Le mécanisme d'héritage autorise leur transmission. La relation d'héritage est définie sur les arbres élémentaires sous-spécifiés qui constituent une grammaire. La sous-spécification peut concerner la couleur des branches, la catégorie étiquetant les sommets ou bien un trait. Chaque arbre hérite des propriétés des arbres qui sont au-dessus de lui dans la hiérarchie.

En figure 5, la hiérarchie du groupe prépositionnel est représentée de façon schématique. Chaque cellule contient un arbre élémentaire. 


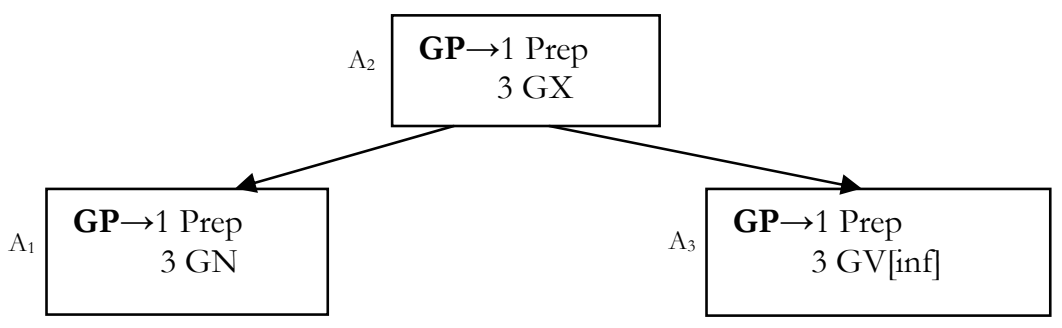

Figure 5

En $A_{2}$, la catégorie qui est en position 3 dans le groupe prépositionnel est laissée indéterminée (variable GX) car elle peut se réaliser de deux façons : soit en GN, soit en GV[inf].

La hiérarchisation implique une relation d'ordre entre les arbres. C'est une relation de subsomption. On dit que l'arbre $A_{2}$ subsume l'arbre $A_{1}$ lorsque $A_{1}$ est plus spécifié que $A_{2}$. Les constructions les moins spécifiées représentent celles qui sont les plus générales. La relation d'ordre permet d'abstraire les propriétés communes à plusieurs constructions et d'isoler les propriétés qui distinguent les constructions. Ceci permet, dans les GAP, de décrire des propriétés positionnelles indépendamment des catégories qui occupent les positions.

\section{Traitement des constructions infinitives dans les GAPH}

Dans cette seconde partie, l'analyse des constructions infinitives est représentée dans le cadre des grammaires d'arbres polychromes hiérarchisées. Nous distinguons le cas où les infinitives sont introduites par un verbe conjugué, du cas où elles sont introduites par des prépositions.

Dans son étude sur les constructions infinitives, Huot (1981) distingue deux types de prépositions. D'une part, de, qui selon les cas, est une préposition qui introduit un groupe prépositionnel ou bien un COMP qui est un introducteur de phrase. Et, d'autre part, toutes les autres prépositions qui peuvent introduire un infinitif.

Nous distinguons également deux cas parmi les prépositions, mais nous regroupons quant à nous $\grave{a}$ avec $d e$, mettant à part toutes les autres prépositions.

\subsection{L'infinitif suit immédiatement un verbe conjugué}

(1) Jean veut partir

(2) a. Jean entend chanter les oiseaux / b. Jean entend les oiseaux chanter

(3) Jean a entendu sonner

(4) Jean a entendu chanter le temps des cerises

Ces exemples possèdent tous un verbe à l'infinitif mais ce qui varie c'est le sujet de cet infinitif. En effet, en (1) le sujet de partir est Jean. Jean est également le sujet du 
verbe conjugué veut. Le sujet de l'infinitif est syntaxiquement présent. En (2), le sujet de l'infinitif est distinct du sujet du verbe conjugué : chanter a pour sujet les oiseaux et entend a pour sujet Jean. En (3) et (4), le sujet de sonner et de chanter ne se trouve pas, matériellement dans la phrase. C'est la théorie du contrôle (voir Baschung, 1991), qui s'occupe des principes qui permettent de sélectionner un constituant comme étant le sujet de l'infinitive. Le terme contrôle désigne le processus d'attribution de référence à l'argument implicite de l'infinitive et le contrôleur désigne tout GN interprété comme argument implicite de l'infinitive.

Dans le cas des GAP, cependant, le placement des constituants s'organise selon des positions qui sont définies indépendamment de ce qui les occupe. La structuration arborescente est par conséquent indifférente à la recherche du sujet des infinitives. C'est ainsi que les exemples (1) à (4) s'analysent de la même manière : les infinitives sont considérées comme des phrases. La règle qui les introduit est :

$$
\text { GV } \rightarrow 3 \text { GV } 5 \text { S[inf]. }
$$

Les GV des phrases (1) et (3) ont pour représentation arborescente 6a et le GV de la phrase (2b) 6b. L'infinitive de la phrase (2a) est considérée comme une phrase à sujet inversé, elle a pour représentation $6 \mathrm{c}$. La phrase (4) a la même représentation arborescente que la phrase (2a). Les phrases (2a) et (4) sont différenciées par les traits ${ }^{6}$ qui indiquent les fonctions du GN.

$\mathrm{GV}$

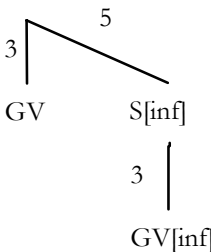

veut $\operatorname{partir}^{7} /$

a entendu sonner

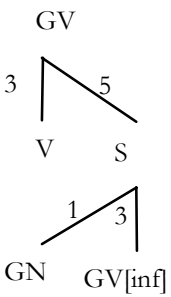

entend les oiseaux chanter

b

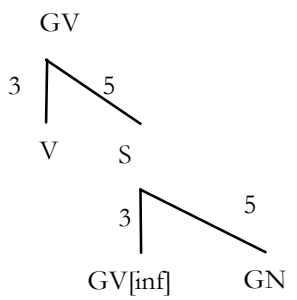

entend chanter les oiseaux/ entend chanter le temps des cerises c

Figure 6

Les exemples (2a) et (4) illustrent l'utilisation de la syntaxe positionnelle. En effet, en (2a) le GN les oiseaux est le sujet de l'infinitif chanter. Et en (4), le GN le temps des cerises est le complément d'objet de l'infinitif chanter (on ne peut pas avoir *Jean a

\footnotetext{
${ }^{6}$ Les traits dans la figure 6 ne sont pas représentés afin de souligner l'aspect unifié des représentations.

${ }^{7}$ On admet la règle $\mathrm{S} \rightarrow 3 \mathrm{GV}$ afin de pouvoir traiter notamment les phrases où le sujet est réalisé comme un clitique, ou les impératives.
} 
entendu chanter le temps des cerises Paul). On voit, ici, qu'une même position peut être occupée par un même élément n'ayant pas la même fonction.

\subsection{L'infinitif est précédé de à ou de}

Les constructions infinitives sont souvent en relation avec un élément qui est soit un verbe, soit un nom, soit un adjectif. Elles sont reliées à cet élément par une préposition (en général de ou a). Huot (1981), dans son étude sur les constructions infinitives du français, distingue de qui se réalise comme un complémenteur quand il précède un verbe à l'infinitif - alors qu'il se réalise comme une préposition quand il précède un nom - de à qui serait toujours une préposition.

Nous allons montrer, à l'aide des tests syntaxiques ci-dessous, pourquoi à notre sens, s'il est juste d'analyser de comme un complémenteur, il n'y a pas de raison de traiter $a ̀$ différemment.

- La substitution
(5a) Jean promet de venir
(5a) *Jean promet de sa venue
(6a) Jean demande à partir
(6b) *Jean demande à son départ
(7a) Jean pense à partir
(7b) Jean pense à son départ
(8a) Jean parle de partir
(8b) Jean parle de son départ

Dans les exemples (7a) et (8a) le verbe à l'infinitif commute avec le groupe nominal, alors qu'en (5a) et (6a) c'est impossible. Ce sont les tours de venir et à partir qui doivent être substitués, c'est-à-dire la préposition et le verbe à l'infinitif.

$$
\begin{aligned}
& \text { Jean promet sa venue } \\
& \text { Jean demande son départ }
\end{aligned}
$$

L'infinitive, qu'elle soit en de ou en à, se substitue par conséquent soit au complément d'objet direct, soit au complément d'objet indirect.

\section{- La reprise pronominale}
(5c) Jean le promet
(et non *en promet)
(6c) ?Jean le demande
(*y demande)
(7c) Jean y pense
(8c) Jean en parle

Dans les exemples (5c) et (6c), le pronom le, qui remplace habituellement les COD, reprend le groupe de +inf. Tandis que dans les exemples (7c) et (8c), à partir et de partir sont repris par les pronoms $y$ et en. Avec $y$, en est le seul pronom qui correspond à la pronominalisation d'un groupe prépositionnel entier, c'est-à-dire de la préposition et de l'élément que celle-ci introduit.

Ce test ne fait que confirmer le test qui précède, en ce qui concerne la fonction de complément direct ou indirect assurée par l'infinitive en de ou en $\grave{a}$. 


\section{- Parallélisme entre infinitive et complétive}
(9) Jean promet qu'il viendra
(10) Jean pense qu'il partira
(10) *Jean parle qu'il partira
(11) *Jean demande qu'il partira

Les exemples (9) et (10), bien que n'ayant pas la même structure de surface, sont équivalents aux exemples (5a) et (7a). Ces derniers acceptent tous deux le parallélisme avec une complétive à verbe non fini.

Ce test montre que certaines infinitives en de ou en à peuvent être remplacées par des complétives. Ce n'est pas la fonction de complément direct ou indirect qui détermine si ce remplacement est possible.

Plus globalement, l'ensemble des tests montre la grande similitude de comportement des infinitives en de et en à, ainsi que leur parenté avec les complétives. C'est pourquoi à et de peuvent être considérés comme des complémenteurs (introduisant des complétives et des relatives).

Les arguments de Huot pour traiter à comme une préposition sont: la très grande majorité des verbes qui sont suivis de à+inf peuvent également être suivis d'un complément nominal et celui-ci est toujours précédé de la préposition $a^{8}$. En cas de détachement à droite, le groupe à+inf est repris tout comme le GN par le pronom $y$.

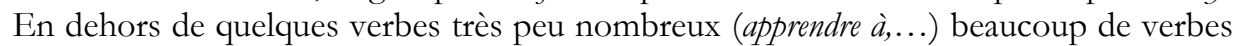
en àtinf mais non suivis de à+ $\mathrm{N}$ ne sont pas nécessairement sans complément nominal. Au contraire la plupart en ont un et celui-ci est toujours prépositionnel.

Mais les exemples suivants montrent que c'est avec les complétives que les infinitives commutent et non avec les GP compléments d'objet indirects.

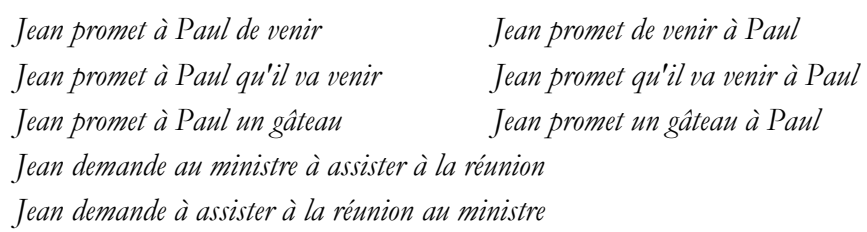

L'analyse que nous proposons consiste par conséquent à intégrer la représentation des infinitives introduites par $d e$, comme celles introduites par $\grave{a}$, dans le traitement des complétives et des relatives présenté plus haut (cf. règle (d)). Nous considérons donc à et de comme des introducteurs de phrase et nous les étiquetons COMP. Par ailleurs, la relation d'interdépendance associée à la règle doit être complétée avec les valeurs à et de pour le type du COMP :

\begin{tabular}{|c|c|c|c|}
\hline T & X & Y & $Z$ \\
\hline de & oui & - & - \\
\hline$\grave{a}$ & oui & - & - \\
\hline
\end{tabular}

On obtient la représentation arborescente de Jean pense à partir (figure $7 \mathrm{~b}$ ).

\footnotetext{
${ }^{8}$ Gross (1975) rassemble dans sa table 7 les verbes à complément indirect en $a$. Seuls une vingtaine seraient, selon lui, suivis de à+inf sans être suivis de à $+\mathrm{N}$.
} 


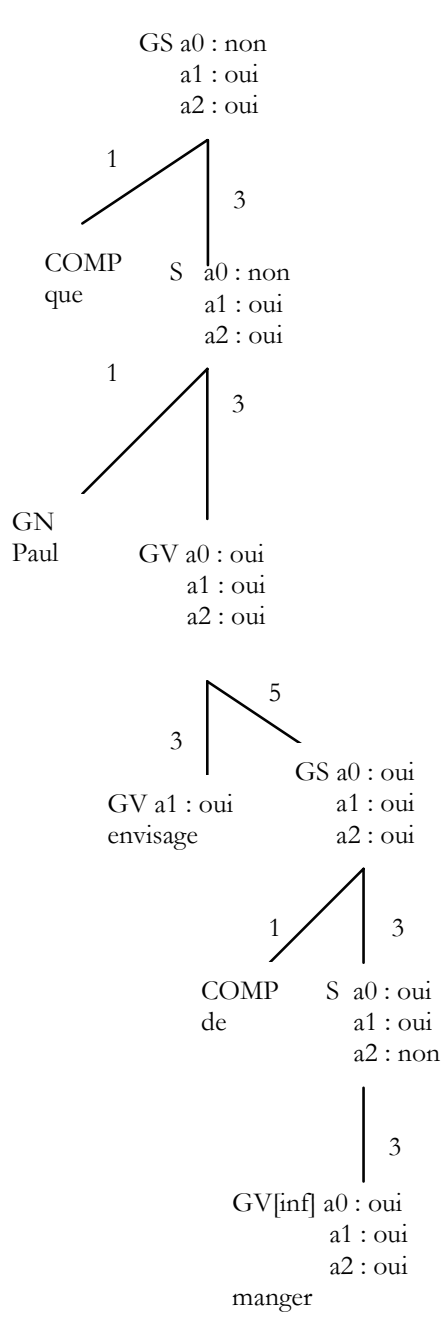

a

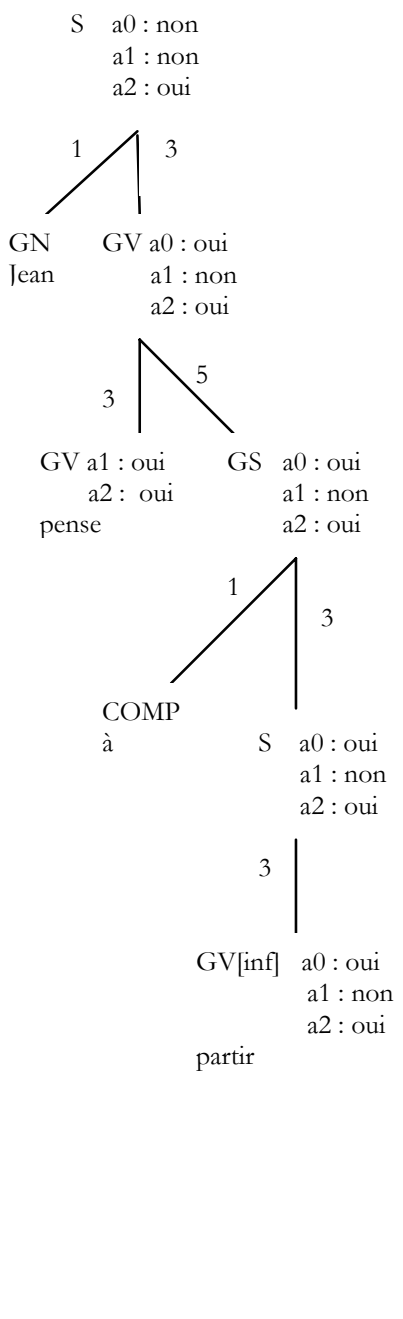

$\mathrm{b}$

Figure 7

Si la proposition infinitive avait été analysée en tant que GP, cela aurait provoqué des difficultés pour une analyse dans le cadre des grammaires d'arbres polychromes. En effet, un GP aurait « rempli » le complément a2 et donc interdit la présence simultanée d'un complément d'objet indirect et d'une proposition infinitive, comme dans Jean promet à Paul de partir.

Inversement la solution proposée permet d'interdire *Jean demande le professeur à assister à la réunion, tout en autorisant Jean demande au ministre à assister à la réunion. 
Un avantage supplémentaire de cette analyse est qu'elle permet un traitement (unifié) des dépendances à distance. Par exemple (la pomme) que Paul envisage de manger (figure 7a).

Grâce à l'analyse de de en introducteur de phrase, la transmission des traits s'effectue correctement: le trait a1 se transmet de nœud en nœud. Le nœud GS participe à la transmission du a1. C'est lui qui transmet le trait au niveau supérieur et non le GV qui se situe à son niveau.

Ce traitement des dépendances à distance est un argument supplémentaire pour considérer à comme un COMP. La représentation de (la voiture) que Paul s'apprête à acheter est en effet l'arbre de la figure (7a) ci-dessus. Si on avait considéré à comme une préposition, un tel traitement aurait été impossible, le groupe prépositionnel ne transmettant pas de trait au groupe verbal dans lequel il est inclus.

\subsection{L'infinitive est introduite par une préposition autre que à et de}

(12) Jean est arrivé sans prévenir

(13) Jean boit pour oublier ses malbeurs

(14) Jean a voté pour élire le nouveau président

On observe que les infinitives introduites par ces prépositions ne peuvent pas avoir la fonction de complément direct. En revanche, elles peuvent avoir la fonction de complément indirect (14) ou de modifieur : (12) et (13).

Mais, dans les deux cas, la dépendance à distance est impossible :

* Le malheur que Jean boit pour oublier

* Le nowveau président que Jean a voté pour élire.

Ces exemples ne peuvent pas recevoir la même analyse que celle des infinitives en à et de. Sans prévenir, pour oublier ses malbeurs et pour élire le nouveau président sont, par conséquent, analysés comme des groupes prépositionnels ${ }^{9}$. Comme on l'a vu (figure 5), un groupe prépositionnel peut être composé d'une préposition et d'un GV. Le GV est obligatoirement au mode infinitif.

\subsection{La biérarchisation $d u G V$}

On donne à titre d'illustration la hiérarchie des réalisations du groupe verbal qui est représentée en figure 8.

\footnotetext{
${ }^{9}$ Le cas de par nécessiterait une étude particulière. Cet item est sous-catégorisé par un nombre très limité de verbes, mais semble admettre la dépendance à distance : l'bistoire que Jean a commencé par raconter.
} 
Les constructions infinitives dans les grammaires d'arbres polychromes hiérarchisées

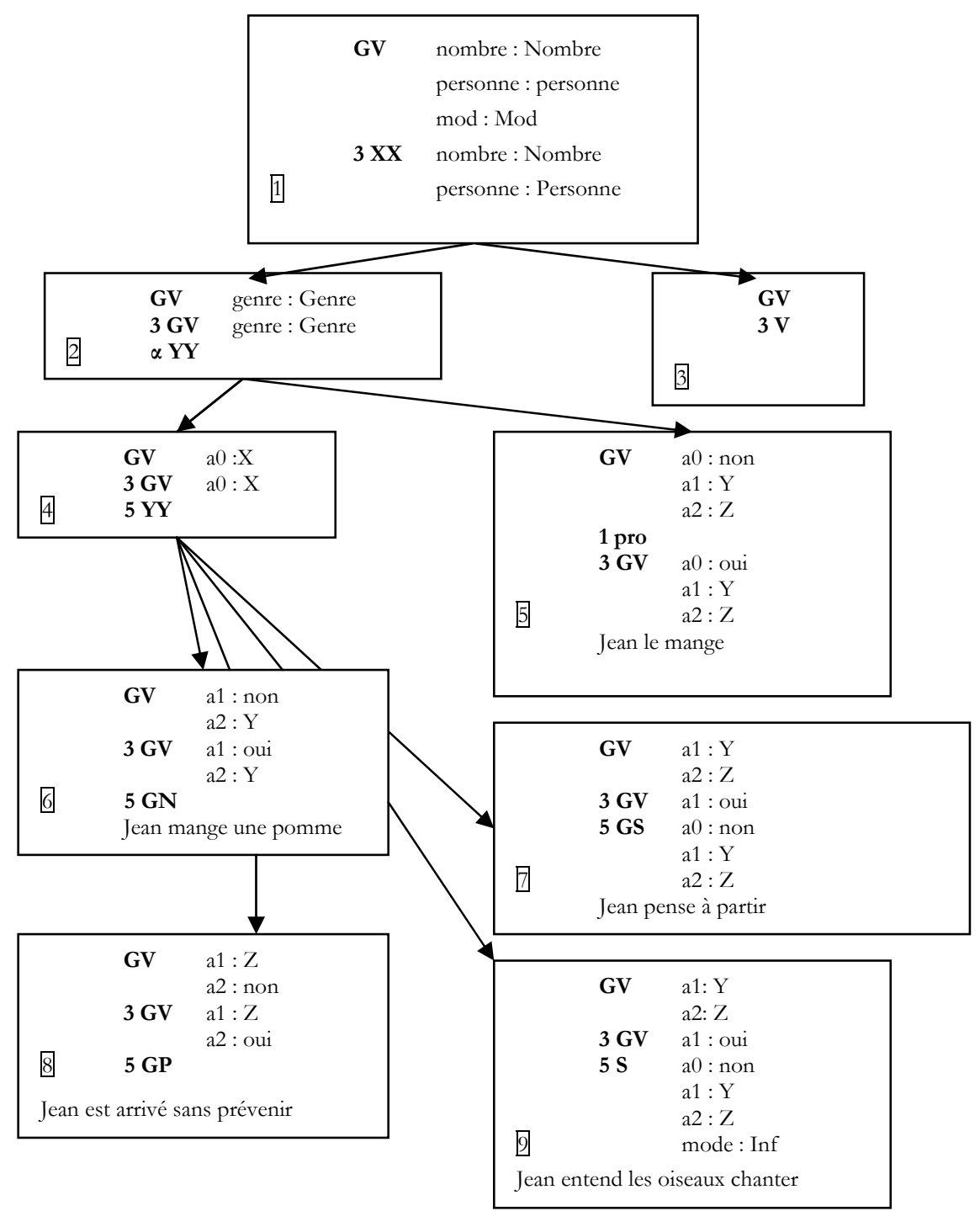

Figure 8

La cellule 1 est «la racine» de toutes les réalisations possibles du GV. La catégorie qui occupe la position 3 n'est pas spécifiée car elle peut se réaliser de deux manières différentes : en GV comme le montre la cellule 2 ou en verbe (cellule 3).

Dans la cellule 2, l'arbre possède une branche dont ni la couleur (variable $\alpha$ ) ni la catégorie (variable YY) n'est spécifiée. En effet, cet arbre peut se réaliser de plusieurs façons : la couleur de la branche prend la valeur 5 dans la cellule 4 et 1 dans la cellule 5 .

La variable YY dans la cellule 4 représente les différentes catégories qui peuvent occuper la position $5 \mathrm{du}$ GV : GN, GS, S, GP. Les cellules 7, 8, 9 sont les représentations des infinitives. 


\section{Conclusion}

Le traitement des constructions infinitives dans les grammaires d'arbres polychromes hiérarchisées a permis d'illustrer les potentialités de ce formalisme. Nous avons vu que l'hypothèse positionnelle permet de donner un traitement unifié des complétives tout en incluant les infinitives. En outre, la relation d'interdépendance conserve les spécificités de ces différentes structures dans le traitement unifié. Et les différentes réalisations du groupe verbal ont pu être regroupées grâce à la hiérarchisation. On a ainsi obtenu « une vue d'ensemble » des réalisations possibles du groupe verbal dans les grammaires d'arbres polychromes.

Anne LABLANCHE

\section{BIBLIOGRAPHIE}

BASCHUNG K., 1991, Grammaires d'unification à traits et contrôles des infinitives en français, ADOSA, Clermont-Ferrand.

BASCHUNG K., 1996, «Une approche lexicalisée des phénomènes de contrôle des infinitives en français ", Langages $n^{\circ} 122$, Larousse, Paris.

CORI M., MARANDIN J.M., 1993, «Grammaires d'arbres polychromes », TAL, vol 34, n¹, pp. 101-132.

CORI M., MARANDIN J.M., 1998, «Héritage de propriétés dans les grammaires d'arbres polychromes », LINX $39 \mathrm{n}^{\circ} 2$, pp. 13-42.

DAELEMANS W., GAZDAR G., De SMEDT K., 1992, «Inheritance in natural language processing », Computational Linguistics $18 \mathrm{n}^{\circ} 2$, pp. 205-218.

FRASER N., et HUDSON R., 1992, Inheritance in word grammar, Computational Linguistics 18 $\mathrm{n}^{\circ} 2$, pp. 133-158.

GREVISSE M., 1993, Le bon usage, 13ème édition, Duculot, Louvain-la-neuve.

GROSS M., 1968, Grammaire transformationnelle du français. Syntaxe du verbe, Larousse, Paris.

GROSS M., 1975, Méthodes en syntaxe, Hermann, Paris.

HUOT H., 1981, Constructions infinitives du français : le subordonnant de, Droz, Paris-Genève.

KERLEROUX F., 1990, «Du mode de l'existence de l'infinitif substantivé en français contemporain », Cahiers de grammaire n 15 , Université de Toulouse-Le Mirail, pp. 57-99.

LEEMAN D., 2002, La phrase complexe, les subordinations, Duculot, Louvain-la-neuve.

LEGRAND G., 1999, "L'étude de la subordonnée infinitive dans les grammaires de référence ", Fonctions syntaxiques et rôles sémantiques, Artois Presses Université, Arras.

LORIAN A., 1962, « La proposition infinitive en français moderne », Vox romanica, pp. 285-294.

MILNER J.C., 1989, Introduction à une science du langage, Seuil, Paris. 